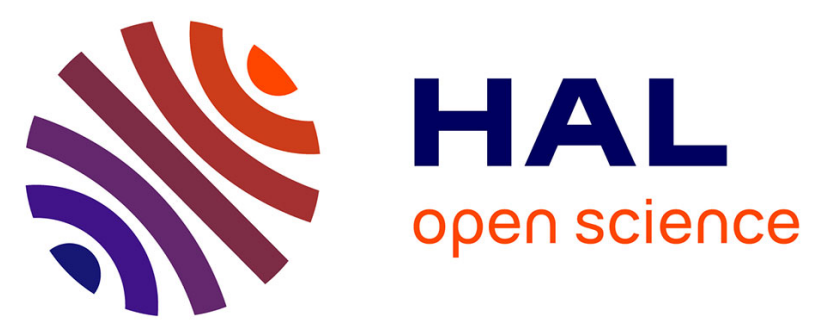

\title{
Time-Varying Spectrum Estimation of Uniformly Modulated Processes by Means of Surrogate Data and Empirical Mode Decomposition
}

\author{
Azadeh Moghtaderi, Patrick Flandrin, Pierre Borgnat
}

\section{- To cite this version:}

Azadeh Moghtaderi, Patrick Flandrin, Pierre Borgnat. Time-Varying Spectrum Estimation of Uniformly Modulated Processes by Means of Surrogate Data and Empirical Mode Decomposition. IEEE International Conference on Acoustics, Speech, and Signal Processing ICASSP-10, Mar 2010, Dallas, Texas, United States. ensl-00476007

\section{HAL Id: ensl-00476007 https://hal-ens-lyon.archives-ouvertes.fr/ensl-00476007}

Submitted on 23 Apr 2010

HAL is a multi-disciplinary open access archive for the deposit and dissemination of scientific research documents, whether they are published or not. The documents may come from teaching and research institutions in France or abroad, or from public or private research centers.
L'archive ouverte pluridisciplinaire HAL, est destinée au dépôt et à la diffusion de documents scientifiques de niveau recherche, publiés ou non, émanant des établissements d'enseignement et de recherche français ou étrangers, des laboratoires publics ou privés. 


\title{
TIME-VARYING SPECTRUM ESTIMATION OF UNIFORMLY MODULATED PROCESSES BY MEANS OF SURROGATE DATA AND EMPIRICAL MODE DECOMPOSITION
}

\author{
Azadeh Moghtaderi, Patrick Flandrin and Pierre Borgnat \\ École Normale Supérieure de Lyon, Laboratoire de Physique \\ 46 allée d'Italie 69364 Lyon Cedex 07, France
}

\begin{abstract}
We propose a new estimate of the time-varying spectra of uniformly modulated processes. The estimate is based on a resampling scheme which incorporates empirical mode decompositions and surrogate data techniques. The performance of the method is studied via simulations.
\end{abstract}

Index Terms - Bandlimited signals, spectral analysis, stochastic processes, time-frequency analysis

\section{INTRODUCTION}

Estimation of the time-varying spectra (TVS) of nonstationary processes remains a challenging problem. This is because (i) many different types of nonstationarity exist and (ii) in contrast with the stationary ergodic case, time averaging cannot be used as a substitute for ensemble averaging to reduce fluctuations in estimates. Although no "universal solution" is expected to exist for all types of nonstationarity, specific approaches can be developed for restricted classes.

In this paper, we focus on the class of uniformly modulated processes (UMPs). Theoretically, UMPs have a simple mathematical form, since they can be decomposed into a stationary process multiplied by a modulating function. More pragmatically, UMPs have been used successfully as models of various real-world processes, e.g., in seismology (see [1], [2] and [3]). We propose here a method for estimating the TVS of a UMP $X$. The method combines an estimate of the TVS obtained by BCMOTIFS estimator introduced in [4] with surrogate data techniques, and proceeds in three steps. The first step estimates the modulating function of $X$ via an empirical mode decomposition. The second step determines the "stationary component" of $\boldsymbol{X}$; surrogate data techniques are then used to create additional "virtual" realizations of $\boldsymbol{X}$. These realizations are then used to compute a more accurate estimate of the TVS of $\boldsymbol{X}$ which is based on the arithmetic averaging of BCMOTIFS estimates for each realization.

In Section 2, we briefly review the UMPs, empirical mode decompositions, and surrogate data techniques. In Sections 3 and 4, we describe in detail the estimation method sketched in the previous paragraph. Finally, in Section 5, we examine the performance of the method on simulated data.

\section{PRELIMINARIES}

\subsection{Uniformly modulated processes}

Let $\boldsymbol{X}=\left\{X_{t}\right\}_{t \in \mathbb{Z}}$ be a discrete-time, $\mathbb{R}$-valued, zero-mean, finite-variance nonstationary process. We say that $X$ is a uniformly modulated process (UMP) if there exists a zeromean stationary process $\boldsymbol{Y}=\left\{Y_{t}\right\}_{t \in \mathbb{Z}}$ with spectrum $S_{\boldsymbol{Y}}$ and a sequence $\left\{C_{t}\right\}_{t \in \mathbb{Z}}$ of positive real numbers, such that $X_{t}=C_{t} Y_{t}$ for each $t \in \mathbb{Z}$. We refer to $C_{t}$ as the modulating function of $X$, and $Y$ is the stationary component of $X$. We assume that the modulating function is "slowly-varying" in comparison with $Y$. More precisely, the modulating function is a bandlimited baseband process while $Y$ is a broadband process without any low-frequency oscillations.

The time-varying spectrum (TVS) [5] of a UMP $X$ is defined by $T_{\boldsymbol{X}}(t, f) \triangleq C_{t}^{2} S_{\boldsymbol{Y}}(f)$ for $(t, f) \in \mathbb{Z} \times[-1 / 2,1 / 2]$.

\subsection{Surrogate data techniques}

Surrogate data techniques comprise a type of resampling technique. Given a single realization of a stochastic process $Y$, surrogate data techniques can produce an arbitrary number of "virtual" realizations of $Y$, called surrogates, with similar statistical properties. Originally, surrogates were used to test for nonlinearity [6]; more recently, they were used in $[7,8]$ to test for nonstationarity. In the latter, the rationale is that, given the same global empirical spectrum, a nonstationary process differs from a stationary one by some structure in time which carries over to the "phase" of the spectrum. Randomizing the phase and keeping the magnitude unchanged leads therefore to a "stationarized" process, while many other realizations can be obtained due to randomization of the phase. In a nonstationary context, this allows the construction of a statistical reference corresponding to the null hypothesis of stationarity. However if the process under study is stationary-as it will be assumed in the following - surrogates can be viewed as virtual realizations.

\subsection{Empirical mode decompositions}

Let $S_{t}$ be an arbitrary signal. Briefly, the empirical mode decomposition (EMD) [9] is a model-free and fully data-driven technique which decomposes $S_{t}$, comprised of superimposed 
oscillations into its zero-mean oscillatory components. This is achieved by a "fine-to-coarse" recursive scheme: The fastest local oscillations (identified through neighbouring local extrema) are subtracted from the signal, yielding a residual signal to which the same procedure can be applied. Extracted in this way, each of the components is referred to as an intrinsic mode function (IMF) of $S_{t}$. The recursion stops when the residual signal has no more oscillation. Denoting IMFs by $M_{t}^{(i)}$ for $1 \leq i \leq I_{\max }$, we write

$$
S_{t}=\sum_{i=1}^{I_{\max }} M_{t}^{(i)}+\rho_{t},
$$

where $\rho_{t}$ is the residual signal. For MATLAB code and further details concerning implementation of the EMD, see [10].

\section{TIME-VARYING SPECTRUM ESTIMATION BY MEANS OF SURROGATE DATA}

Let $X$ be a UMP with modulating function $C_{t}$ and stationary component $Y$. In this section, we describe a general technique to estimate $T_{\boldsymbol{X}}$, based on the use of surrogate data.

Let $\mathscr{X}=X_{0}, X_{1}, \ldots, X_{N-1}$ be a realization of $X$, and let $\widehat{C}_{t}$ be a nonzero estimate of $C_{t}$ for each $t$. Set $\tilde{Y}_{t}=X_{t} / \widehat{C}_{t}$ for each $t$. Provided that each $\widehat{C}_{t}$ estimates $C_{t}$ accurately (see Section 4 for details), $\tilde{\mathscr{Y}}=\tilde{Y}_{0}, \tilde{Y}_{1}, \ldots, \tilde{Y}_{N-1}$ can be regarded as a realization of $\boldsymbol{Y}$. We use $\tilde{\mathscr{Y}}$ to obtain $J$ surrogate data sets $\tilde{\mathscr{Y}}^{j}=\tilde{Y}_{0}^{j}, \tilde{Y}_{1}^{j}, \ldots, \tilde{Y}_{N-1}^{j}$, where $1 \leq j \leq J$.

Define $\tilde{\mathscr{X}}^{j}=\widehat{C}_{0} \tilde{Y}_{0}^{j}, \widehat{C}_{1} \tilde{Y}_{1}^{j}, \ldots, \widehat{C}_{N-1} \tilde{Y}_{N-1}^{j}$, where again $1 \leq j \leq J$. We call $\tilde{\mathscr{X}}^{j}$ the $j$ th nonstationary surrogate data obtained from $\mathscr{X}$ and regard this data as elements of the ensemble of $\boldsymbol{X}$. For each $1 \leq j \leq J$, let $\widehat{T}_{\boldsymbol{X}}^{j}$ be an estimate of $T_{\boldsymbol{X}}$ based solely on $\tilde{\mathscr{X}}^{j}$. We take the arithmetic average of the $\widehat{T}_{\boldsymbol{X}}^{j}$ as an estimate of $T_{\boldsymbol{X}}$, which we call the averaged surrogate-based estimate:

$$
\widehat{T}_{\boldsymbol{X}}^{\mathrm{av}}(t, f) \triangleq \frac{1}{J} \sum_{j=1}^{J} \widehat{T}_{\boldsymbol{X}}^{j}(t, f), \quad(t, f) \in \mathbb{Z} \times[-1 / 2,1 / 2] .
$$

In this paper, we choose each $\widehat{T}_{\boldsymbol{X}}^{j}$ to be the BCMOTIFS estimator $[4,11]$. The rationale behind this choice is as follows: For general processes, theoretical and simulation results in [4] indicate BCMOTIFS has lower bias and variance than other estimators of the TVS, especially near the boundaries of the time-frequency region. In particular, for UMPs, BCMOTIFS is approximately unbiased [4].

\section{MODULATING FUNCTION ESTIMATION}

Let $X$ and $\mathscr{X}$ be as in Section 3, and assume that $X_{t} \neq 0$ for each $t$. To proceed with the technique proposed in Section 3, we must estimate each $C_{t}$. In this section, we describe how $C_{t}$ and $\mathscr{X}$ can be "decoupled," leading to an estimate of $C_{t}$.

Define $X_{t}^{*}=\log \left|X_{t}\right|$, so that $X_{t}^{*}=\log C_{t}+\log \left|Y_{t}\right|$ for each $t$. Write $\mathscr{X}^{*}=\log \left|X_{0}\right|, \log \left|X_{1}\right|, \ldots, \log \left|X_{N-1}\right|$. We can compute the EMD of $\mathscr{X}^{*}$ to obtain its IMFs $M_{t}^{(i)}$, and write $X_{t}^{*}$ as in Eq. (1) when $S_{t}$ is replaced by $X_{t}^{*}$. Note that the applicability of EMD method in general, does not require particular forms of oscillations and therefore appropriate for the log-transformed signal. Loosely speaking, the assumption made in Section 2.1 states that the oscillations of $\log C_{t}$ are much slower than those in $\log \left|Y_{t}\right|$. As a result the oscillations of $\log C_{t}$ are accurately described by a set of high-order IMFs such that for $1 \leq i_{*} \leq I_{\max }$ we have

$$
\log C_{t} \approx \sum_{i=i_{*}}^{I_{\max }} M_{t}^{(i)}+\rho_{t} .
$$

Following above, estimating $C_{t}$ reduces to determining $i_{*}$.

We now describe three approaches to determining $i_{*}$.

The energy of the $i$ th IMF is defined by $E^{(i)}=\sum_{t=0}^{N-1}\left|M_{t}^{(i)}\right|^{2}$ for each $i$. For typical broadband signals, $E^{(i)}$ is decreasing in $i$ [12]. Identifying the smallest index $i+1$ such that $E^{(i+1)}>E^{(i)}$ can therefore provide information about the value of $i_{*}$. This is called the energy approach.

Denote the number of zero crossings of the $i$ th IMF by $Z^{(i)}$. It can be observed that, in the absence of low-frequency oscillations, broadband signals give rise to a family of IMFs satisfying $R^{(i+1)} \approx 2$, where $R^{(i+1)}=Z^{(i)} / Z^{(i+1)}$ whenever it is defined. Furthermore, we assume that the random variables $R^{(i+1)}$ are approximately equally distributed. We call $R^{(i+1)}$ the ith ratio of zero crossing numbers ( $i$ th RZCN). Hence the smallest index $i+1$ for which $R^{(i+1)}$ is "significantly different" from 2 can provide information about the value of $i_{*}$. The question is how to quantify "significantly different." To answer this question, we construct 13 processes (all approximately broadband without low-frequency oscillations), including (i) nine fractional Gaussian noise processes with Hurst exponents $H=0.1,0.2, \ldots, 0.9$, (ii) two $\operatorname{AR}(2)$ processes, and (iii) two nonstationary processes, the first being $\mathrm{AR}(2)$ with time-dependent coefficients and the second being frequency-modulated. For each process, we create 5000 realizations of length $N=1000$, and compute the EMDs and RZCN of each. We then compute the empirical distribution of $\vec{R}=\left[\begin{array}{llll}\vec{R}_{1} & \vec{R}_{2} & \cdots & \vec{R}_{5000}\end{array}\right]$ where denoting the $i$ th RZCN of the $b$ th realization by $R_{b}^{(i+1)}, 1 \leq i \leq I_{\max }^{b}-1$, we have $\vec{R}_{b}=$ $\left[R_{b}^{(2)} R_{b}^{(3)} \cdots R_{b}^{\left(I_{\max }^{b}\right)}\right]$ for $1 \leq b \leq 5000$. Fig. 1 shows the empirical distribution of $\vec{R}$ computed for each process and shown in different colors. The result of our simulations encourages the idea that, regardless of the type of broadband process, the distribution of $\vec{R}$ remains unchanged. As a result of this, we may then propose a common threshold test for determining when $R^{(i+1)}$ is significantly different from 2 . For each process, we compute the empirical distribution of $R^{(i+1)}$ over all the realizations and for each $i=1,2 \ldots, I_{\max }-1$ respectively where here we assume $I_{\max }=10$. In this case, if, as an example, for a particular realization, we have $I_{\max }=8$, we assume $R^{(8)}=R^{(9)}=R^{(10)}$ and if $I_{\max }=12$, we discard $R^{(11)}$. We then calculate $5 \%$ and $95 \%$ significance level 


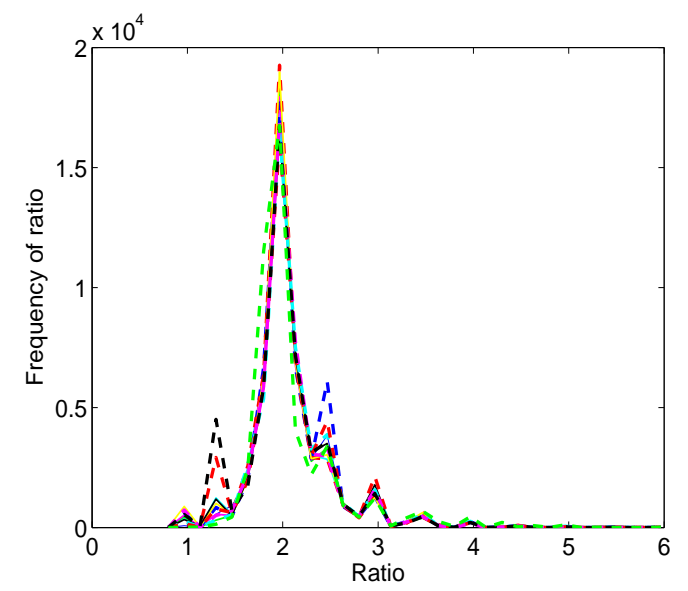

Fig. 1. Empirical distribution of $\vec{R}$ computed for different processes (different colors). Apart from the expected peak at 2 , there exist several smaller but visible peaks. They appear in the presence of high order IMFs which have small values of zero crossing numbers.

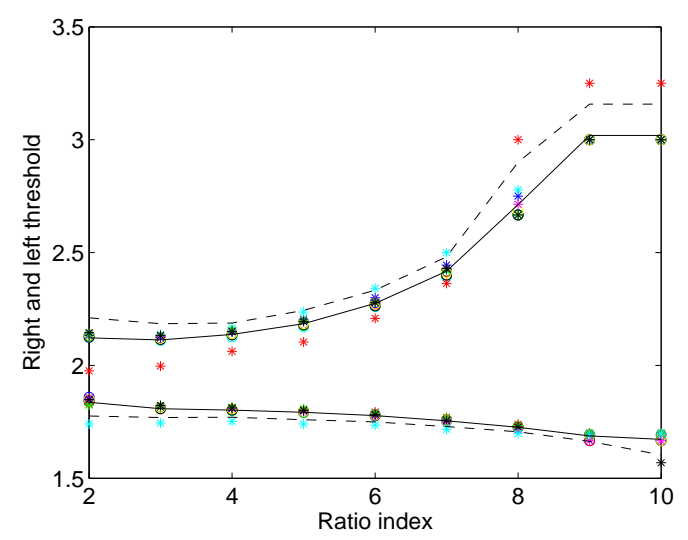

Fig. 2. The right and left thresholds (marked by stars) computed for different processes (different colors). Solid lines indicate averaged left and right thresholds and dashed lines indicate two standard deviation of the averaged thresholds.

of each distribution, and refer to them as ith right and left thresholds respectively. Fig. 2 shows the right and left thresholds for each process (different colors). Any RZCN which is outside of the two standard deviation of the averaged $i$ th left and right thresholds within 13 process is significantly different from 2. Finally, as mentioned earlier, the smallest index $i+1$ where $R^{(i+1)}$ is significantly different from 2 determines $i_{*}$. The problem is that since the selection of the left and right thresholds are entirely based on an empirical result, it is always possible that the smallest $i+1$ is a false detection and not the correct $i_{*}$. This approach is called the ratio approach.

The energy and ratio approaches can be combined in order to reduce the number of false detects as follows: For each $1 \leq i \leq I_{\max }-1$, we compute each index $i+1$ such that $E^{(i+1)}>E^{(i)}$. We also evaluate every index $i+1$ where $R^{(i+1)}$ is significantly different from 2 . We then choose $i_{*}$ to be the smallest common index in both approaches. The value of $i_{*}$ is then plugged into Eq. (2) in order to evaluate $\log C_{t}$. The estimated modulating function is $\widehat{C}_{t}=\exp \left(\log C_{t}\right)$. This combined approach is called the energy-ratio approach.

\section{NUMERICAL EXAMPLES}

Let $\boldsymbol{X}^{(k)}=\left\{X_{t}^{(k)}\right\}_{t \in \mathbb{Z}}, 1 \leq k \leq 5$ be UMPs with modulating functions

$$
\begin{array}{ccc}
C_{t}^{(1)} & =\mathrm{e}^{-\frac{(t-500)^{2}}{2(200)^{2}}}, \quad C_{t}^{(2)} & =\sqrt{1+t / T} \\
C_{t}^{(3)} & =2+\sin (2 \pi p t), \quad C_{t}^{(4)} & =1.5+\cos (2 \pi q t) \\
C_{t}^{(5)}=2-\mathrm{e}^{-\frac{(t-500)^{2}}{2(200)^{2}}} &
\end{array}
$$

where $T=200, p=0.002$ and $q=0.001$. The stationary components of the $\boldsymbol{X}^{(k)}$ are as follows: $\boldsymbol{Y}^{(1)}, \boldsymbol{Y}^{(2)}$, and $\boldsymbol{Y}^{(3)}$ are fractional Gaussian noise with Hurst parameters $H=0.2$, $H=0.5$, and $H=0.8$, respectively, and $\boldsymbol{Y}^{(4)}$ and $\boldsymbol{Y}^{(5)}$ are the $\mathrm{AR}(2)$ processes

$$
\begin{aligned}
& Y_{t}^{(4)}=0.2 Y_{t-1}^{(4)}+0.5 Y_{t-2}^{(4)}+\zeta_{t} \\
& Y_{t}^{(5)}=0.8 Y_{t-1}^{(5)}-0.4 Y_{t-2}^{(5)}+\epsilon_{t} .
\end{aligned}
$$

Here, $\left\{\zeta_{t}\right\}_{t \in \mathbb{Z}}$ and $\left\{\epsilon_{t}\right\}_{t \in \mathbb{Z}}$ are independent white noise processes with variance $10^{4}$.

For each $k$, we create 5000 realizations of length $N=$ 1000 of $\boldsymbol{X}^{(k)}$ and compute the EMD for each realization. We then apply the energy, ratio, and energy-ratio approaches to each realization and each UMP to evaluate $i_{*}$, denoted $i_{*, a}(k)$, where $a=1,2,3$ indicate energy, ratio and energyratio approaches respectively. Using our theoretical knowledge of the modulating function for each UMP, we pick $i_{*}$ which gives the minimum $L^{2}$-distance between $\log C_{t}$ from Eq. (2) and theoretical log modulating function. We denote $i_{*}$ obtained from minimum $L^{2}$-distance by $i_{\dagger}(k)$. Table 1 shows the number of times in 5000 where we have obtained $i_{*, a}(k)=i_{\dagger}(k), i_{*, a}(k)=i_{\dagger}(k)+1, i_{*, a}(k)=$ $i_{\dagger}(k)-1$, or $\left|i_{*, a}(k)-i_{\dagger}(k)\right|>1$. It is clear from Table 1 that the energy-ratio approach outperforms the other two approaches. We now take $\boldsymbol{X}^{(4)}$ for further analysis. Let $\mathscr{X}=X_{0}, X_{1}, \ldots, X_{N-1}$ be a realization of $\boldsymbol{X}^{(4)}$ and $\mathscr{X}^{*}$ the log-transform of $\mathscr{X}$. We compute the EMD of $\mathscr{X}^{*}$ to obtain its IMFs and then evaluate $E^{(i)}$ and $R^{(i+1)}$ for each $i$. Fig. 3 shows the plot of $E^{(i)}, 1 \leq i \leq I_{\max }$, (top plot) and $R^{(i+1)}, 1 \leq i \leq I_{\max }-1$, (bottom plot) where $I_{\max }=9$ in this example. Applying the energy approach we determine that there are 4 indexes $i+1$ where $i=3,5,7,8$ which satisfies $E^{(i+1)}>E^{(i)}$. On the other hand, applying the ratio approach, we find out that there are three indexes $i+1$ for $i=6,7,8$ where $R^{(i+1)}$ is significantly different from 2 . These indexes are marked by red triangles in Fig 3. Applying the energy-ratio approach, we see that the first common index between the two approaches is $i_{*}=8$.

Using $i_{*}=8$, we evaluate $\widehat{C}_{t}$ (see Section 4 ). The estimate $\widehat{C}_{t}$ is then used to create $J=50$ nonstationary surrogate 


\begin{tabular}{|l|c|c|c|}
\hline & $\begin{array}{c}\text { Energy } \\
a=1\end{array}$ & $\begin{array}{c}\text { Ratio } \\
a=2\end{array}$ & $\begin{array}{c}\text { Energy-Ratio } \\
a=3\end{array}$ \\
\hline$i_{*, a}(1)=i_{\dagger}(1)$ & 2361 & 1926 & 3193 \\
$i_{*, a}(1)=i_{\dagger}(1)+1$ & 1353 & 918 & 717 \\
$i_{*, a}(1)=i_{\dagger}(1)-1$ & 583 & 484 & 891 \\
$\left|i_{*, a}(1)-i_{\dagger}(1)\right|>1$ & 703 & 1672 & 199 \\
\hline$i_{*, a}(2)=i_{\dagger}(2)$ & 1866 & 1420 & 2697 \\
$i_{*, a}(2)=i_{\dagger}(2)+1$ & 1181 & 735 & 948 \\
$i_{*, a}(2)=i_{\dagger}(2)-1$ & 658 & 495 & 949 \\
$\left|i_{*, a}(2)-i_{\dagger}(2)\right|>1$ & 1295 & 2350 & 406 \\
\hline$i_{*, a}(3)=i_{\dagger}(3)$ & 1919 & 1339 & 2710 \\
$i_{*, a}(3)=i_{\dagger}(3)+1$ & 1386 & 902 & 985 \\
$i_{*, a}(3)=i_{\dagger}(3)-1$ & 373 & 521 & 910 \\
$\left|i_{*, a}(3)-i_{\dagger}(1)\right|>1$ & 1322 & 2238 & 395 \\
\hline$i_{*, a}(4)=i_{\dagger}(4)$ & 2636 & 1893 & 3316 \\
$i_{*, a}(4)=i_{\dagger}(4)+1$ & 1100 & 980 & 643 \\
$i_{*, a}(4)=i_{\dagger}(4)-1$ & 232 & 492 & 740 \\
$\left|i_{*, a}(4)-i_{\dagger}(4)\right|>1$ & 1032 & 1635 & 301 \\
\hline$i_{*, a}(5)=i_{\dagger}(5)$ & 2615 & 1487 & 3598 \\
$i_{*, a}(5)=i_{\dagger}(5)+1$ & 1056 & 902 & 637 \\
$i_{*, a}(5)=i_{\dagger}(5)-1$ & 295 & 363 & 529 \\
$\left|i_{*, a}(5)-i_{\dagger}(5)\right|>1$ & 1034 & 2248 & 236 \\
\hline
\end{tabular}

Table 1. Comparison between energy, ratio and energy-ratio approaches using the theoretical $\log C_{t}$ for 5 UMPs.
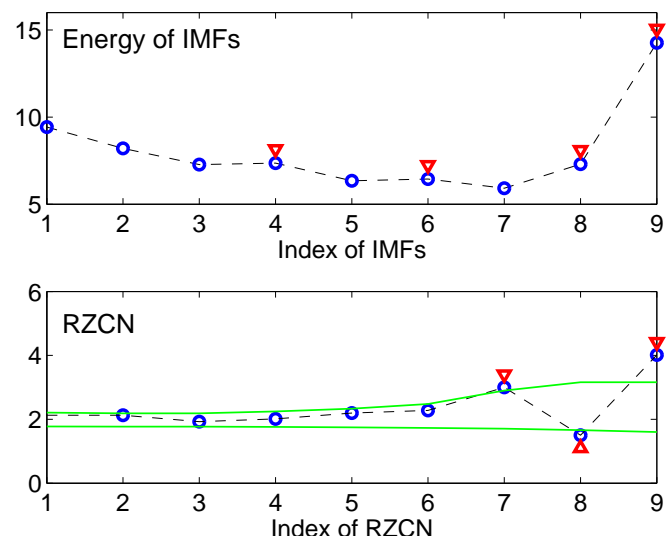

Fig. 3. $E^{(i)}, i=1,2, \ldots, 9$ (top) and $R^{(i+1)}, i=1,2, \ldots, 8$ (bottom). The red triangles mark the indexes which satisfy the conditions in energy (top) and ratio (bottom) approaches.

data $\tilde{\mathscr{X}}^{j}, 1 \leq j \leq 50$ (see Section 3). Using 50 nonstationary surrogate data, we then estimate $T_{\boldsymbol{X}}$ using the averaged surrogate-based estimate $\widehat{T}_{\boldsymbol{X}}^{\text {av }}$ (see Section 3). Fig. 4 shows the performance of this estimator in comparison with the BCMOTIFS estimator for $\mathscr{X}$.

\section{CONCLUSION}

In this paper, we proposed a new scheme to estimate the timevarying spectra of uniformly modulated processes. The estimate proceeds by computing "virtual" realizations, using surrogate data and empirical mode decompositions. Simulation results suggest that the new estimator performs well, and affords a significant improvement over BCMOTIFS.
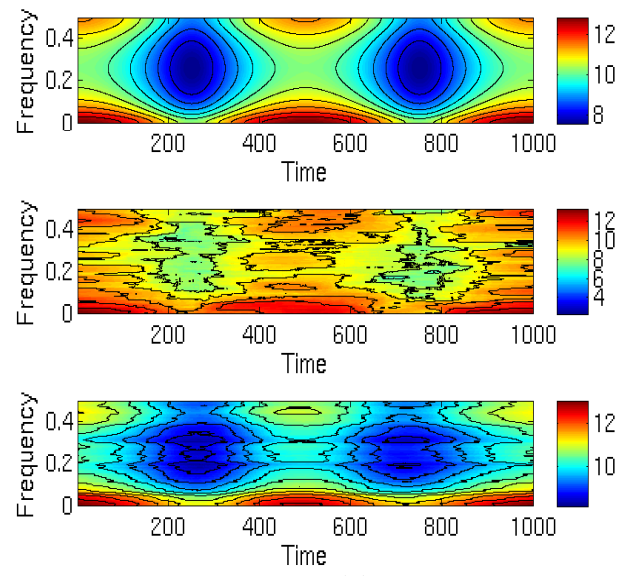

Fig. 4. Theoretical TVS of $\boldsymbol{X}^{(4)}$ (top), estimate of the TVS using $\mathscr{X}$ (middle), estimate of the TVS using $\tilde{\mathscr{X}}^{j}, 1 \leq 1 \leq$ 50 (bottom). The parameters used in BCMOTIFS estimator are $N W=4, K=7$ and $B=201$.

\section{REFERENCES}

[1] L. J. Herbst, "Periodogram analysis and variance fluctuation," Journal of the Royal Statistical Society, Series B, vol. 25, pp. 442-450, 1963.

[2] T. Fujita and H. Shibara, "On a model of earthquake ground motions for response analysis and some examples of analysis through experiment," Conference on Engineering Design for Earthquake Environments, vol. 12, pp. 139-148, 1978.

[3] G. R. Dargahi-Noubary and P. J. Laycock, "Special ratio discriminant and information theory," Journal of Time Series Analysis, vol. 2, no. 2 , pp. 71-85, 1981 .

[4] A. Moghtaderi, Multitaper Methods for Time-Frequency Spectrum Estimation and Unaliasing of Harmonic Frequencies, Ph.D. thesis, Queen's University, 2009.

[5] G. Mélard and A. H. Schutter, "Contributions to evolutionary spectral theory," Journal of Time Series Analysis, vol. 10, no. 1, pp. 41-63, 1989.

[6] J. Theiler, S. Eubank, A. Longtin, B. Galdrikian, and J. D. Farmer, "Testing for nonlinearity in time series: the method of surrogate data," Physica D, vol. 58, no. 1-4, pp. 77-94, 1992.

[7] J. Xiao, P. Borgnat, and P. Flandrin, "Testing stationarity with timefrequency surrogates," in Proceedings of EUSIPCO-07, Poznań, Poland, 2007, pp. 2020-2024.

[8] J. Xiao, P. Borgnat, P. Flandrin, and C. Richard, "Testing stationarity with surrogates-a one-class SVM approach," in Proceedings of the IEEE Statistical Signal Processing Workshop (SSP-07), Madison, WI, 2007, pp. 720-724.

[9] N. E. Huang, Z. Shen, S. R. Long, M. L. Wu, H. H. Shih, Q. Zheng, N. C. Yen, C. C. Tung, and H. H. Liu, "The empirical mode decomposition and Hilbert spectrum for nonlinear and non-stationary time series analysis," Proceedings of the Royal Society of London A: Mathematical, Physical and Engineering Sciences, vol. 454, pp. 903-995, 1998.

[10] G. Rilling, P. Flandrin, and P. Gonçalves, "On empirical mode decomposition and its algorithms," in IEEE-EURASIP Workshop on Nonlinear Signal and Image Processing (NSIP-03), 2003.

[11] A. Moghtaderi, G. Takahara, and D. J. Thomson, "Evolutionary spectrum estimation for uniformly modulated processes with improved frequency resolution," in Proceedings of the IEEE Statistical Signal Processing Workshop (SSP-09), 2009, pp. 765-768.

[12] P. Flandrin, G. Rilling, and P. Gonçalves, "Empirical mode decomposition as a filter bank," IEEE Signal Processing Letters, vol. 11, no. 2 pp. 112-114, 2004. 